Research Article

\title{
Prevalence of Anemia in Pediatric IBD Patients and Impact on Disease Severity: Results of the Pediatric IBD-Registry CEDATA-GPGE ${ }^{\circledR}$
}

\author{
Jan de Laffolie, ${ }^{1}$ Martin W. Laass, ${ }^{2}$ Dietmar Scholz, ${ }^{3}$ Klaus-Peter Zimmer, ${ }^{1}$ \\ Stephan Buderus, ${ }^{4}$ and CEDATA-GPGE Study Group ${ }^{5}$ \\ ${ }^{1}$ General Pediatrics \& Neonatology, Justus-Liebig-University, Giessen, Germany \\ ${ }^{2}$ Department of Pediatrics, Medical Faculty Carl Gustav Carus, Technische Universität Dresden, Dresden, Germany \\ ${ }^{3}$ Department of Child and Adolescent Psychiatry, Psychotherapy and Psychosomatics, University of Leipzig, Leipzig, Germany \\ ${ }^{4}$ Pediatric and Adolescent Medicine, GFO Kliniken Bonn, St. Marien-Hospital, Bonn, Germany \\ ${ }^{5}$ Gesellschaft für pädiatrische Gastroenterologie und Ernährung, Chausseestr 128-129, Berlin, Germany
}

Correspondence should be addressed to Jan de Laffolie; jan.delaffolie@paediat.med.uni-giessen.de

Received 10 May 2017; Accepted 8 June 2017; Published 5 December 2017

Academic Editor: Amosy M'Koma

Copyright (C) 2017 Jan de Laffolie et al. This is an open access article distributed under the Creative Commons Attribution License, which permits unrestricted use, distribution, and reproduction in any medium, provided the original work is properly cited.

Aim. To determine the prevalence of anemia and its association with disease severity in children and adolescents with IBD. Methods. CEDATA-GPGE is a registry for pediatric patients with IBD in Germany and Austria from 90 specialized centers. As markers of disease severity, analysis included patient self-assessment on a Likert scale $(1-5 ; 1=$ very good) and physicians' general assessment $(0=$ no activity to $4=$ severe disease) and the disease indices. Anemia was defined as hemoglobin concentration below the 3rd percentile. Results. Prevalence of anemia was $65.2 \%$ in CD and $60.2 \%$ in UC. Anemic CD and UC patients showed significantly worse self-assessment than patients without anemia (average \pm standard deviation; CD: $3.0 \pm 0.9$ versus $2.5 \pm 0.9, p<0.0001$; UC: $2.9 \pm 0.9$ versus $2.3 \pm 0.9, p<0.0001$ ). Accordingly, physicians' general assessment (PGA) was significantly worse in anemic than in nonanemic patients in CD $(p<0.0001)$ and UC $(p<0.0001)$. PCDAI in anemic CD, $p<0.0001$, and PUCAI in anemic UC patients, $p<0.0001$, were significantly higher than in nonanemic patients. $40.0 \%$ of anemic CD and $47.8 \%$ of anemic UC patients received iron during follow-up. Conclusion. Almost 2/3 of pediatric IBD patients are anemic. Patients' self-assessment and disease severity as determined by PGA and activity indices are worse in anemic patients. Contrastingly, only a minority received iron therapy.

\section{Introduction}

Pediatric onset inflammatory bowel disease (IBD) is a group of chronic, relapsing disorders of the gastrointestinal tract, which affect an increasing number of children and adolescents. While incidence in adult populations seems to stabilize, pediatric onset is increasing. In recent decades, there has been a general increase in the incidence and prevalence of IBD in both industrialized and emerging countries [1]. A shift towards earlier onset of IBD has been observed within pediatrics [2]. Recent studies indicate incidence rates of 5 to 11 per 100,000 children under the age of 18 years [3]. About
$20 \%$ of all IBD patients are diagnosed before the age of 18 years, and early onset disease is associated with a high degree of patient morbidity, missed work or school, decreased quality of life, and psychosocial issues [4-7]. Anemia is a common problem in IBD patients. In pediatric IBD, anemia is reported in up to $75 \%$ of patients $[8,9]$. The description of various phenotypic presentations in pediatric IBD and a higher rate of anemic patients has also led to the notion of anemia being an important extraintestinal manifestation of IBD $[7,10]$.

Anemia can occur in pediatric IBD patients for different reasons: gastrointestinal blood loss, insufficient dietary intake 
of iron, decreased absorption of dietary iron, and decreased activity of bone marrow because of chronic inflammation [11]. Anemia potentially leads to significant reduction in health-related quality of life, impairs physiological development, growth, and psychosocial processes [12], and poses a significant economic burden on health systems [13].

This interferes with the aims of therapy in pediatric IBD: relief of symptoms, normal growth and pubertal development, improved quality of life, but minimization of drug toxicity [14]. Diagnosis and adequate treatment of iron deficiency anemia is important, especially since previous studies have shown that duration of anemia can be reduced by iron supplementation [15].

Previous studies on anemia in pediatric IBD included relatively small patient numbers [16]. The aim of this study is to provide prevalence data of anemia and iron treatment in the, to date, largest cohort of pediatric IBD patients at time of diagnosis and after one to five years of their disease. We also explored the impact of anemia on subjective and objective measurements of disease activity and subjective general well-being.

\section{Methods}

CEDATA-GPGE is a registry, currently based at the University Children's Hospital Giessen for pediatric IBD patients ( 0 -18 years of age) in German-speaking countries from over 90 pediatric gastroenterology centers in Germany and Austria [7]. The database included all patient data sets with diagnosis from 2004 until July 2010. Inclusion criteria comprised of at least one follow-up within 3 months of the first report to the registry. This period of time (time of diagnosis until the first follow-up) will subsequently be referred to as "at diagnosis." To further improve data quality, only patients with at least two follow-up visits within one year following diagnosis were included. Data were analyzed retrospectively for the time of diagnosis and during follow-up (mean 103 days, range 1-1645 days).

Anemia was defined as hemoglobin below the 3rd percentile for age and gender in recent population-based pediatric $\mathrm{Hb}$ reference values in Germany as published by the KiGGS Study (Table 1). To evaluate the clinical significance of anemia, severe anemia was defined as a concentration of hemoglobin more than $2 \mathrm{~g} / \mathrm{dl}$ below the $3 \mathrm{rd}$ percentile for age and gender [17].

Patient self-assessment was quantified on a Likert scale from 1 to 5 ( 1 = very good and $5=$ very bad). Disease activity was measured by physicians' general assessment on a 4 point scale ( $1=$ no activity/remission and $4=$ severe activity) and by calculation of the Pediatric Crohn's Disease Activity Index (PCDAI) for patients with $\mathrm{CD}$ and the Pediatric Ulcerative Colitis Activity Index (PUCAI) [18, 19]. The hematocrit in PCDAI and rectal bleeding in PUCAI were excluded, since they are directly related to bleeding and anemia to calculate a modified PCDAI or PUCAI, respectively, at time of diagnosis.

Prevalence of anemia were calculated once at the time of diagnosis and subsequently in a cross section fashion in all patients after one to five years follow-up of their disease course. The registry does not hold data on ferritin or transferrin. A decrease in mean corpuscular volume (MCV) was considered as suggestive for iron deficiency anemia. Since azathioprine can increase MCV, azathioprine therapy was compared in patients with anemia and without anemia to correct for potential bias.

2.1. Statistical Methods. Analysis was performed in SAS (Cary, NC, USA) and R (The R Foundation for Statistical Computing, Vers 3.0.2). Some of the graphics and tables were redrawn in Microsoft Excel. Distribution was assessed for normality, and $t$-test was performed when comparing continuous or pseudonormal data; otherwise, Mann-Whitney $U$ Test was performed where appropriate. Statistical significance was assumed when $p<0.05$. The database and reporting procedure were examined and approved by the relevant ethics committees and data protection employees of the three institutions that maintained the registry during the time period covered by the research (the Institute for Medical Informatics and Biometry (IMB) of Technische Universität Dresden; the Institute of Epidemiology at the Helmholtz Zentrum, LMU Munich; and the Department of General Pediatrics and Neonatology, JLU Giessen). The Austrian hospitals that participated also obtained local ethics approval.

\section{Results}

3554 patients were initially included in the study, with a total of 25,765 patient contact documentations. 109 patients were excluded because of incomplete initial data, 51 for missing date of primary diagnosis, 22 for implausible data or typing errors, and 616 patients for missing the second follow-up. 2756 patients (1753 CD, 882 UC, and 121 IBD-U (IBD unclassified)) were available for analysis.

Of 2756 children and adolescents with IBD, 1743 (63.2\%) suffered from anemia at any time during the disease course-referred to standard values for hemoglobin from the KiGGS study (Table 1). Anemia was slightly more frequent in CD $(65.2 \%)$ than in UC $(60.2 \%, p=0.013)$ and in IBD-U $(61.8 \%, p=0.049)$.

Anemic CD patients assessed their own well-being significantly worse than $C D$ patients without anemia (3.0 \pm 0.9 versus $2.5 \pm 0.9$, mean \pm standard deviation, $p<0.0001)$. Similar significant differences were found in anemic versus nonanemic UC patients $(2.9 \pm 0.9$ versus $2.3 \pm 0.9$, mean \pm standard deviation (SD), $p<0.0001)$ and in anemic versus nonanemic IBD-U (median 3.0, interquartile range (IQR) versus median 2.0, IQR; $p<0.001$ Mann-Whitney $U$ test) (Figure 1).

The physicians' general assessment was significantly worse in anemic than in nonanemic patients in CD $(2.7 \pm 0.8$ versus $2.1 \pm 0.9, p<0.0001)$, UC $(2.6 \pm 0.9$ versus $2.0 \pm 0.8, p<0.0001$ ), and IBD-U (median 2.5 versus 2.0, IQR 1; $p=0.0003 \mathrm{MWU})$. In all three disease groups, anemic patients had higher disease activity with a median between low and medium activity (Figure 2).

Anemic CD patients showed a significantly higher PCDAI $(27.1 \pm 14.1$ versus $18.7 \pm 12.0$, mean \pm SD,$p<0.0001)$ than nonanemic, PUCAI was significantly higher in anemic versus 
TABLE 1: Hemoglobin concentration reference values (in $\mathrm{g} / \mathrm{dl}$ ) from the German KiGGS study.

\begin{tabular}{|c|c|c|c|c|c|c|c|c|c|}
\hline $\begin{array}{l}\text { Age } \\
\text { (yrs.) }\end{array}$ & P3 & P5 & P10 & P25 & $\begin{array}{c}\text { P50 } \\
\text { (median) }\end{array}$ & P75 & P90 & P95 & P97 \\
\hline \multicolumn{10}{|c|}{ Boys $(n=7205)$} \\
\hline 1,5 & 10,1 & 10,3 & 10,6 & 11,1 & 11,6 & 12,1 & 12,6 & 12,8 & 13,0 \\
\hline 2 & 10,3 & 10,5 & 10,7 & 11,2 & 11,7 & 12,2 & 12,7 & 13,0 & 13,1 \\
\hline 2,5 & 10,4 & 10,6 & 10,9 & 11,4 & 11,9 & 12,4 & 12,8 & 13,1 & 13,2 \\
\hline 3 & 10,5 & 10,7 & 11,0 & 11,5 & 12,0 & 12,5 & 12,9 & 13,2 & 13,4 \\
\hline 3,5 & 10,6 & 10,8 & 11,1 & 11,6 & 12,1 & 12,6 & 13,0 & 13,3 & 13,5 \\
\hline 4 & 10,8 & 10,9 & 11,2 & 11,7 & 12,2 & 12,7 & 13,1 & 13,4 & 13,5 \\
\hline 4,5 & 10,8 & 11,0 & 11,3 & 11,8 & 12,3 & 12,8 & 13,2 & 13,5 & 13,6 \\
\hline 5 & 10,9 & 11,1 & 11,4 & 11,8 & 12,3 & 12,8 & 13,3 & 13,5 & 13,7 \\
\hline 5,5 & 11,0 & 11,2 & 11,5 & 11,9 & 12,4 & 12,9 & 13,3 & 13,6 & 13,8 \\
\hline 6 & 11,1 & 11,3 & 11,5 & 12,0 & 12,5 & 13,0 & 13,4 & 13,7 & 13,8 \\
\hline 6,5 & 11,1 & 11,3 & 11,6 & 12,0 & 12,5 & 13,0 & 13,5 & 13,7 & 13,9 \\
\hline 7 & 11,2 & 11,4 & 11,6 & 12,1 & 12,6 & 13,1 & 13,5 & 13,8 & 13,9 \\
\hline 7,5 & 11,2 & 11,4 & 11,7 & 12,1 & 12,6 & 13,1 & 13,6 & 13,8 & 14,0 \\
\hline 8 & 11,3 & 11,5 & 11,7 & 12,2 & 12,7 & 13,2 & 13,6 & 13,9 & 14,0 \\
\hline 8,5 & 11,3 & 11,5 & 11,8 & 12,2 & 12,7 & 13,2 & 13,7 & 13,9 & 14,1 \\
\hline 9 & 11,4 & 11,6 & 11,8 & 12,3 & 12,8 & 13,3 & 13,7 & 14,0 & 14,2 \\
\hline 9,5 & 11,4 & 11,6 & 11,9 & 12,3 & 12,8 & 13,3 & 13,8 & 14,0 & 14,2 \\
\hline 10 & 11,5 & 11,7 & 11,9 & 12,4 & 12,9 & 13,4 & 13,8 & 14,1 & 14,3 \\
\hline 10,5 & 11,5 & 11,7 & 12,0 & 12,4 & 13,0 & 13,5 & 13,9 & 14,2 & 14,4 \\
\hline 11 & 11,6 & 11,8 & 12,0 & 12,5 & 13,0 & 13,5 & 14,0 & 14,3 & 14,4 \\
\hline 11,5 & 11,6 & 11,8 & 12,1 & 12,6 & 13,1 & 13,6 & 14,1 & 14,4 & 14,5 \\
\hline 12 & 11,7 & 11,9 & 12,2 & 12,7 & 13,2 & 13,7 & 14,2 & 14,5 & 14,7 \\
\hline 12,5 & 11,8 & 12,0 & 12,3 & 12,7 & 13,3 & 13,8 & 14,3 & 14,6 & 14,8 \\
\hline 13 & 11,9 & 12,1 & 12,4 & 12,9 & 13,4 & 14,0 & 14,5 & 14,8 & 15,0 \\
\hline 13,5 & 12,0 & 12,2 & 12,5 & 13,0 & 13,6 & 14,2 & 14,7 & 15,0 & 15,3 \\
\hline 14 & 12,1 & 12,3 & 12,7 & 13,2 & 13,8 & 14,4 & 15,0 & 15,3 & 15,5 \\
\hline 14,5 & 12,3 & 12,5 & 12,8 & 13,4 & 14,0 & 14,6 & 15,2 & 15,6 & 15,8 \\
\hline 15 & 12,5 & 12,7 & 13,0 & 13,6 & 14,2 & 14,9 & 15,5 & 15,8 & 16,0 \\
\hline 15,5 & 12,7 & 12,9 & 13,3 & 13,8 & 14,5 & 15,1 & 15,7 & 16,1 & 16,3 \\
\hline 16 & 12,9 & 13,1 & 13,5 & 14,0 & 14,7 & 15,3 & 15,9 & 16,3 & 16,5 \\
\hline 16,5 & 13,1 & 13,3 & 13,6 & 14,2 & 14,9 & 15,5 & 16,1 & 16,5 & 16,8 \\
\hline 17 & 13,2 & 13,5 & 13,8 & 14,4 & 15,0 & 15,7 & 16,3 & 16,7 & 17,0 \\
\hline 17,5 & 13,4 & 13,6 & 13,9 & 14,5 & 15,2 & 15,9 & 16,5 & 16,9 & 17,2 \\
\hline \multicolumn{10}{|c|}{ Girls $(n=6870)$} \\
\hline 1,5 & 10,3 & 10,5 & 10,8 & 11,3 & 11,9 & 12,4 & 12,8 & 13,1 & 13,2 \\
\hline 2 & 10,4 & 10,6 & 10,9 & 11,4 & 11,9 & 12,4 & 12,9 & 13,1 & 13,3 \\
\hline 2,5 & 10,5 & 10,7 & 11,0 & 11,5 & 12,0 & 12,5 & 12,9 & 13,2 & 13,3 \\
\hline 3 & 10,6 & 10,8 & 11,1 & 11,5 & 12,1 & 12,5 & 13,0 & 13,2 & 13,4 \\
\hline 3,5 & 10,7 & 10,8 & 11,1 & 11,6 & 12,1 & 12,6 & 13,0 & 13,3 & 13,4 \\
\hline 4 & 10,7 & 10,9 & 11,2 & 11,7 & 12,2 & 12,7 & 13,1 & 13,3 & 13,5 \\
\hline 4,5 & 10,8 & 11,0 & 11,3 & 11,8 & 12,3 & 12,7 & 13,2 & 13,4 & 13,6 \\
\hline 5 & 10,9 & 11,1 & 11,4 & 11,8 & 12,3 & 12,8 & 13,2 & 13,5 & 13,6 \\
\hline 5,5 & 11,0 & 11,2 & 11,4 & 11,9 & 12,4 & 12,9 & 13,3 & 13,5 & 13,7 \\
\hline 6 & 11,0 & 11,2 & 11,5 & 12,0 & 12,4 & 12,9 & 13,3 & 13,6 & 13,7 \\
\hline 6,5 & 11,1 & 11,3 & 11,6 & 12,0 & 12,5 & 13,0 & 13,4 & 13,6 & 13,8 \\
\hline 7 & 11,2 & 11,4 & 11,6 & 12,1 & 12,6 & 13,0 & 13,4 & 13,7 & 13,8 \\
\hline
\end{tabular}

Table 1: Continued.

\begin{tabular}{lccccccccc}
\hline $\begin{array}{l}\text { Age } \\
\text { (yrs.) }\end{array}$ & P3 & P5 & P10 & P25 & $\begin{array}{c}\text { P50 } \\
\text { (median) }\end{array}$ & P75 & P90 & P95 & P97 \\
\hline 7,5 & 11,2 & 11,4 & 11,7 & 12,1 & 12,6 & 13,1 & 13,5 & 13,7 & 13,9 \\
8 & 11,3 & 11,5 & 11,7 & 12,2 & 12,7 & 13,1 & 13,5 & 13,8 & 13,9 \\
8,5 & 11,4 & 11,5 & 11,8 & 12,2 & 12,7 & 13,2 & 13,6 & 13,8 & 14,0 \\
9 & 11,4 & 11,6 & 11,9 & 12,3 & 12,8 & 13,2 & 13,6 & 13,9 & 14,0 \\
9,5 & 11,5 & 11,6 & 11,9 & 12,3 & 12,8 & 13,3 & 13,7 & 14,0 & 14,1 \\
10 & 11,5 & 11,7 & 11,9 & 12,4 & 12,9 & 13,4 & 13,8 & 14,0 & 14,2 \\
10,5 & 11,5 & 11,7 & 12,0 & 12,4 & 12,9 & 13,4 & 13,8 & 14,1 & 14,2 \\
11 & 11,6 & 11,8 & 12,0 & 12,5 & 13,0 & 13,5 & 13,9 & 14,1 & 14,3 \\
11,5 & 11,6 & 11,8 & 12,1 & 12,5 & 13,0 & 13,5 & 14,0 & 14,2 & 14,4 \\
12 & 11,6 & 11,8 & 12,1 & 12,6 & 13,1 & 13,6 & 14,0 & 14,3 & 14,4 \\
12,5 & 11,6 & 11,8 & 12,1 & 12,6 & 13,1 & 13,6 & 14,1 & 14,3 & 14,5 \\
13 & 11,6 & 11,8 & 12,1 & 12,6 & 13,1 & 13,6 & 14,1 & 14,4 & 14,5 \\
13,5 & 11,6 & 11,8 & 12,1 & 12,6 & 13,1 & 13,7 & 14,1 & 14,4 & 14,6 \\
14 & 11,6 & 11,8 & 12,1 & 12,6 & 13,2 & 13,7 & 14,2 & 14,4 & 14,6 \\
14,5 & 11,6 & 11,8 & 12,1 & 12,6 & 13,2 & 13,7 & 14,2 & 14,5 & 14,7 \\
15 & 11,5 & 11,7 & 12,1 & 12,6 & 13,2 & 13,7 & 14,2 & 14,5 & 14,7 \\
15,5 & 11,5 & 11,7 & 12,0 & 12,6 & 13,2 & 13,7 & 14,2 & 14,5 & 14,7 \\
16 & 11,5 & 11,7 & 12,0 & 12,6 & 13,2 & 13,7 & 14,3 & 14,6 & 14,8 \\
16,5 & 11,4 & 11,6 & 12,0 & 12,6 & 13,2 & 13,8 & 14,3 & 14,6 & 14,8 \\
17 & 11,4 & 11,6 & 12,0 & 12,5 & 13,2 & 13,8 & 14,3 & 14,6 & 14,8 \\
17,5 & 1,3 & 11,6 & 11,9 & 12,5 & 13,1 & 13,8 & 14,3 & 14,6 & 14,9 \\
\hline & & & & & & & & &
\end{tabular}

nonanemic UC patients $(36.1 \pm 24.7$ versus $19.1 \pm 20.4$, $p<0.0001)$.

The Pearson correlation coefficient for disease activity indices (PCDAI or PUCAI, resp.) and hemoglobin concentration in $\mathrm{CD}$ patients and UC patients was moderately negative with -0.66 and -0.45 ( $p=0.0057 ; p=0.0173)$.

The prevalence of anemia during follow-up in the three IBD groups for the first 5 years is displayed in Figure 3. Noticeable is the decrease in prevalence of anemia in UC during the first five years of disease in comparison to a relatively unchanged high prevalence in $\mathrm{CD}$.

Average MCV was significantly below normal in all patient groups (see Tables 2 and 3 reference values from KiGGS study). MCV was significantly lower in patients without azathioprine than in patients with azathioprine (regardless of anemia): $\mathrm{CD}$ ( $n=956$ with azathioprine versus 798 without azathioprine), $77.29 \pm 7.54$ versus $81.67+-7.76$; UC $(n=550$ versus 331$), 80.06 \pm 7.53$ versus $84.24 \pm 7.76$; and IBD-U ( $n=90$ versus 31$), 77.27 \mathrm{fl} \pm 7.51$ versus $84.27 \mathrm{fl}$ $\pm 6.28, p<0.0001$ for all). $60.1 \%$ of patients on azathioprine and $65.3 \%$ without azathioprine presented with anemia $(p=0.003$, OR $0.79(0.67-0.9295 \% \mathrm{CI}))$. While $38 \%$ of anemic patients on azathioprine had low MCV, the share in patients without azathioprine was 56\% $(p<0.001$, OR 0.48 (0.39-0.59)). When correcting for an estimated $+4 \mathrm{fl}$ effect of azathioprine on $\mathrm{MCV}$, this difference was not significant ( $57 \%$ versus $56 \%$ ), so the residual share of microcytic anemia, a correlate of iron deficiency anemia, can be estimated at about $50 \%$. The correlation between MCV and azathioprine 
Self-assessed general well-being in pediatric IBD patients

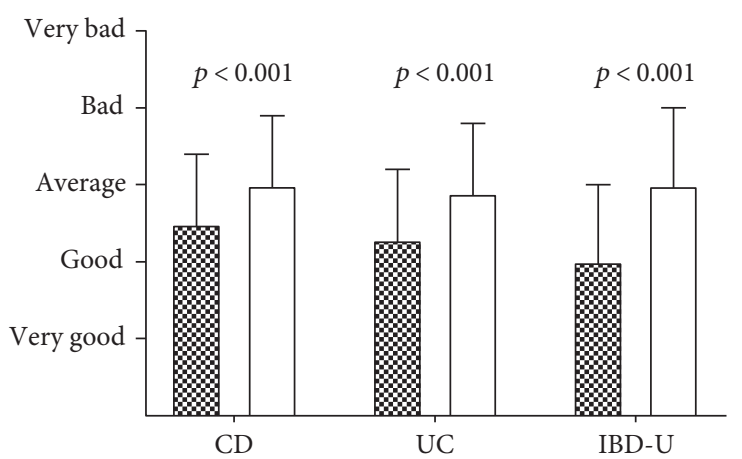

FIGURE 1: Self-assessment of general well-being of pediatric IBD patients comparing anemic (white) and nonanemic (filled bars) patients (mean $\pm \mathrm{SD}$ where parametric, median interquartile range (IQR) where nonparametric) ( $n=\mathrm{CD} 1144 / 609$; UC 531/351; CI $68 / 53)$.

dosage was weak to moderate depending on group selection $(r=0.14-0.30)$.

A subgroup analysis was performed for potential correlation of anemia with severity of disease and age group. In the youngest age group (patients $<6$ years of age), $46 \%$ of $\mathrm{CD}$, $53 \%$ of UC, and $38 \%$ of IBD-U patients were anemic.

In the group 6 to $<10$ years, $60 \%$ of $C D$ patients, $61 \%$ UC patients, and $42 \%$ of IBD-U patients showed anemia. In the oldest age group $\geq 10$ years, $C D$ patients were anemic in $67 \%$ of the cases, UC in $61 \%$, and IBD-U in 58\%. Anemia prevalence was significantly higher in adolescents with $\mathrm{CD}$ in comparison to $\mathrm{CD}$ patients $<6$ years $(p=0.0005$, OR 2.34 (95\% CI 1.45-3.79)), and all other differences were not significant.

Of all anemic pediatric IBD patients, 94\% had mild and $6 \%$ severe anemia. In all age groups, mild anemia was noted in $63.5 \%$ of CD patients, $54.6 \%$ of UC patients, and $46.6 \%$ of IBD-U patients and severe anemia in $2.1 \%, 5.8 \%$, and $5.3 \%$, respectively. In the patients $<6$ years of age, $42.3 \%$ of CD patients suffered from mild and $4.2 \%$ from severe anemia, while $41.4 \%$ of UC patients had mild and $12.1 \%$ severe anemia (IBD-U 33.3\% and 4.7\%).

$56.9 \%$ of CD patients from the middle age group (6 to $<10$ years) suffered from mild anemia, 3.6\% from severe, and in UC, the percentage was $54.4 \%$ and $6.4 \%$ (IBD-U $38.4 \%$ and $3.8 \%)$. The adolescents $\geq 10$ years of age had a mild anemia in $65.3 \%$ and severe in $1.8 \%$ when CD was the diagnosis, $55.7 \%$ and $5.1 \%$ when UC was the diagnosis, and $52.4 \%$ and $5.9 \%$ when IBD-U was the diagnosis, respectively.

Only 740 (42.5\%) of 1743 anemic patients received iron therapy at the time anemia was present and documented. While in UC, a larger population of female anemic patients received iron supplementation compared to their male counterparts (52.2\% (145) versus 43.1\% (109), $p=0.0365)$, and this trend did not reach statistical significance in CD or IBD-U (43\% (196) versus 38.1\% (262); 46.2\% (12) versus $38.1 \%(16)$ ). Data on route of iron supplementation (oral or parenteral) or dosing regiments were not recorded in
Physician's general assessment in pediatric IBD patients



Figure 2: Physicians' general assessment of disease activity in anemic (white) versus nonanemic (filled bars) patients sorted by diagnosis ( $n=$ CD 1144/609; UC 531/351; CI 68/53).

the registry. $\mathrm{CD}$ and $\mathrm{UC}$ patients with severe anemia received iron supplementation more often than patients with mild anemia: $41 \%$ versus $18.9 \%$ of UC patients, $22.2 \%$ versus $14.4 \%$ of CD patients and $20.4 \%$ versus $20 \%$ of IBD-U patients.

\section{Discussion}

We found in our study that almost two-thirds of pediatric IBD patients were anemic at the time of diagnosis. So far, there are very few studies that aim at anemia and iron deficiency in pediatric IBD, most of them in rather small cohorts and with short follow-up $[8,9,15,16]$.

Previously reported data from smaller studies have shown similar prevalence of anemia with $70 \%$ in children and $42 \%$ in adolescents compared to $40 \%$ in adults $[9,16]$.

At presentation, we found a relatively high percentage of anemic patients (all IBD 63.2\%, CD 65.5\%, UC 60.4\%, and IBD-U 51.9\%), but less than in other studies reported like the $75 \%$ observed by Wiskin et al. [8].

When comparing age groups 0 to $<6$ years, 6 to $<10$ years, and $\geq 10$ years, we found an increase in anemia rate at presentation with age (CD $46.5 \%$ versus $60.5 \%$ versus $67.1 \%$; UC $53.4 \%$ versus $60.8 \%$ versus $61.0 \%$; IBD-U $38.1 \%$ versus $42.3 \%$ versus $58.3 \%$ ). However, the proportion of severe anemic patients decreases (CD $4.2 \%$ versus $3.6 \%$ versus $1.8 \%$; UC $12.1 \%$ versus $6.4 \%$ versus $5.2 \%$; IBD-U $4.8 \%$ versus $3.8 \%$ versus $5.9 \%$ ). A potential explanation could be the prolonged diagnostic latency in early and very early onset IBD that has been described previously for the German population [20].

Wiskin et al. also reported that $30 \%$ of patients with pediatric IBD are anemic two years after their initial diagnosis [8]. In our population, prevalence of anemia two years after the initial diagnosis was higher for CD with $44.8 \%$, but comparable for UC (36.31\%) and IBD-U (33.3\%). This confirms the relatively high rates found by Gerasimidis et al. with $65 \%$ after one year [16].

Looking at the long-term data, in CD patients the anemia rate remains surprisingly stable at about $50 \%$, while prevalence decreases for UC (from $53.4 \%$ at diagnosis to $37.9 \%$ 


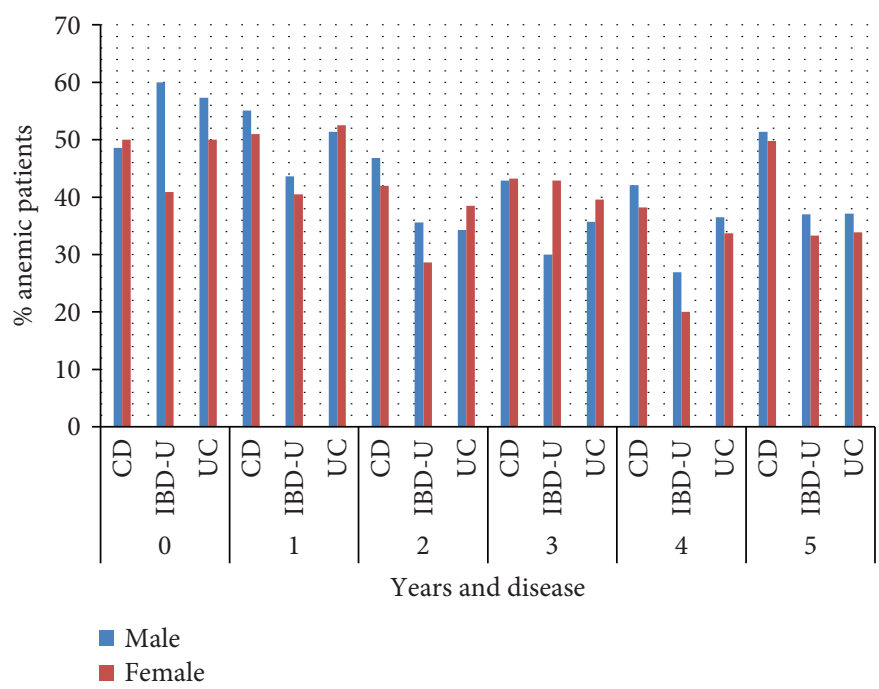

Figure 3: Prevalence of anemia in the first five years of disease by disease and gender ( $n$ at time points are: $0=654 ; 1=1664,2=1559$; $3=1293 ; 4=1022 ; 5=1114)$.

TABle 2: Hematological results of Crohn's disease patients with anemia (mean $\pm \mathrm{SD})$. Hb: hemoglobin concentration; MCV: mean corpuscular volume; Hct: hematocrit; m: male; f: female.

\begin{tabular}{lcccc}
\hline $\begin{array}{l}\text { Time point } \\
\text { (years after } \\
\text { diagnosis) }\end{array}$ & & $\mathrm{Hb}(\mathrm{g} / \mathrm{dl})$ & $\mathrm{MCV}(\mathrm{fl})$ & $\mathrm{Hct}(\%)$ \\
\hline 0/at diagnosis & $\mathrm{m}(n=122)$ & $10.9 \pm 1.3$ & $72.9 \pm 6.0$ & $34.7 \pm 4.7$ \\
& $\mathrm{f}(n=91)$ & $10.0 \pm 1.2$ & $73.0 \pm 7.4$ & $32.4 \pm 3.5$ \\
\hline \multirow{2}{*}{1} & $\mathrm{~m}(n=337)$ & $11.8 \pm 1.5$ & $78.7 \pm 7.7$ & $36.1 \pm 5.3$ \\
& $\mathrm{f}(n=227)$ & $11.2 \pm 1.5$ & $78.6 \pm 7.8$ & $34.4 \pm 3.8$ \\
\hline \multirow{2}{*}{3} & $\mathrm{~m}(n=273)$ & $11.7 \pm 1.5$ & $79.1 \pm 8.0$ & $35.2 \pm 2.9$ \\
& $\mathrm{f}(n=177)$ & $11.0 \pm 1.5$ & $80.2 \pm 8.3$ & $34.5 \pm 5.4$ \\
\hline \multirow{3}{*}{4} & $\mathrm{~m}(n=211)$ & $11.8 \pm 1.2$ & $79.7 \pm 8.0$ & $36.0 \pm 4.1$ \\
& $\mathrm{f}(n=143)$ & $10.8 \pm 1.4$ & $80.0 \pm 8.0$ & $34.1 \pm 3.2$ \\
\hline \multirow{2}{*}{5} & $\mathrm{~m}(n=167)$ & $12.0 \pm 1.5$ & $79.6 \pm 8.8$ & $36.4 \pm 4.1$ \\
& $\mathrm{f}(n=99)$ & $11.0 \pm 1.1$ & $78.9 \pm 8.3$ & $34.3 \pm 4.2$ \\
\hline & $\mathrm{m}(n=203)$ & $12.5 \pm 1.8$ & $81.3 \pm 7.8$ & $38.0 \pm 5.2$ \\
& $\mathrm{f}(n=137)$ & $11.3 \pm 1.4$ & $81.0 \pm 9.3$ & $33.2 \pm 2.1$ \\
\hline
\end{tabular}

after 5 years) and IBD-U (from $50 \%$ at diagnosis to $35.4 \%$ after 5 years).

The impact of anemia on adult patients' quality of life has previously been described. Typical symptoms include not only headache, fatigue, and dizziness but also impairment of cognitive function, stomatitis, and esophageal webs [21]. Our data support the suggested association of anemia with low self-assessed well-being, high disease activity as evaluated by the physician, and higher activity indices, despite correction for obviously anemia-related items, namely, hematocrit in PCDAI and bloody stools and hemoglobin in PUCAI. These items were eliminated in the calculation to avoid a bias towards anemic patients having more inflammatory activity.
TABLE 3: Hematological results of ulcerative colitis patients with anemia (mean $\pm \mathrm{SD}$ ). $\mathrm{Hb}$ : hemoglobin concentration; MCV: mean corpuscular volume; Hct: hematocrit; m: male; f: female.

\begin{tabular}{lcccc}
\hline $\begin{array}{l}\text { Time } \\
\text { (years after } \\
\text { diagnosis) }\end{array}$ & & HB (g/dl) & MCV (fl) & Hct (\%) \\
\hline 0 & $\mathrm{~m}(n=51)$ & $10.1 \pm 1.5$ & $75.5 \pm 7.7$ & $30.9 \pm 5.8$ \\
& $\mathrm{f}(n=45)$ & $9.7 \pm 1.5$ & $77.8 \pm 6.5$ & $31.8 \pm 6.4$ \\
\hline 1 & $\mathrm{~m}(n=129)$ & $11.9 \pm 1.7$ & $80.5 \pm 7.1$ & $36.2 \pm 4.4$ \\
& $\mathrm{f}(n=136)$ & $10.9 \pm 1.7$ & $80.5 \pm 7.7$ & $34.2 \pm 6.0$ \\
\hline \multirow{2}{*}{3} & $\mathrm{~m}(n=87)$ & $10.9 \pm 1.4$ & $79.2 \pm 8.9$ & $34.0 \pm 7.2$ \\
& $\mathrm{f}(n=92)$ & $10.3 \pm 1.2$ & $80.2 \pm 9.0$ & $32.4 \pm 7.2$ \\
\hline \multirow{3}{*}{4} & $\mathrm{~m}(n=74)$ & $11.6 \pm 1.7$ & $80.0 \pm 7.0$ & $35.6 \pm 4.0$ \\
& $\mathrm{f}(n=84)$ & $10.3 \pm 1.3$ & $81.5 \pm 8.5$ & $32.3 \pm 3.5$ \\
\hline \multirow{2}{*}{5} & $\mathrm{~m}(n=58)$ & $11.6 \pm 1.5$ & $81.2 \pm 7.9$ & $36.1 \pm 3.9$ \\
& $\mathrm{f}(n=56)$ & $11.2 \pm 1.3$ & $80.6 \pm 7.1$ & $32.8 \pm 7.2$ \\
\hline & $\mathrm{m}(n=66)$ & $12.0 \pm 2.0$ & $82.6 \pm 8.5$ & $38.2 \pm 4.1$ \\
& $\mathrm{f}(n=74)$ & $11.4 \pm 1.5$ & $82.8 \pm 7.9$ & $35.1 \pm 3.2$ \\
\hline
\end{tabular}

4.1. Iron Deficiency Anemia. Besides the multifactorial etiology of anemia in IBD, the vast majority of anemic IBD patients suffer from iron deficiency anemia $[11,21]$.

Wiskin et al. reported prevalences of iron deficiency in $90 \%$, respectively, $95 \%$ and $70 \%$ in CD and UC patients, respectively, $65 \%$ at follow-up after two years [8].

Our data can only provide indirect information on the etiology of anemia from hemoglobin and MCV, since no parameters of iron metabolism like ferritin, transferrin saturation, or soluble transferrin receptor were obtained by the registry.

Decreased MCV as a surrogate marker for iron deficiency anemia has to be interpreted with caution since medication used in pediatric IBD can significantly change MCV. Several studies have suggested a correlation with 
TABLE 4: Treatment of anemic patients with iron supplementation.

\begin{tabular}{|c|c|c|c|c|c|c|}
\hline \multirow{2}{*}{ Iron treatment } & \multicolumn{2}{|c|}{ Crohn's disease } & \multicolumn{2}{|c|}{ Ulcerative colitis } & \multicolumn{2}{|c|}{ IBD-U } \\
\hline & Male & Female & Male & Female & Male & Female \\
\hline Yes & $262(38.1 \%)$ & $196(43.0 \%)$ & $109(43.1 \%)$ & $145(52.2 \%)$ & $16(38.1 \%)$ & $12(46.2 \%)$ \\
\hline No & $426(61.9 \%)$ & $260(57.0 \%)$ & 144 (56.9\%) & $133(47.8 \%)$ & $26(61.9 \%)$ & $14(53.8 \%)$ \\
\hline
\end{tabular}

6-thioguaninnucleotide (6-TGN) levels, and some even suggested MCV as a surrogate marker instead of measuring metabolites for therapeutic drug monitoring [22].

From our data, correction for azathioprine exposure was difficult to estimate, since metabolism differs significantly between individuals even receiving the same dose of azathioprine or 6-MP [23] and metabolite concentrations were not collected in the registry.

In our patient cohort, we confirmed a significantly higher $\mathrm{MCV}$ in the patient groups with azathioprine compared to patients without, but the difference was less pronounced $(+4 \mathrm{fl}$; data not shown). When corrected for this difference, the residual share of microcytic anemia, therefore estimated share of iron deficiency anemia was around $50 \%$.

4.2. Iron Therapy. Only about $1 / 3$ to half of anemic patients received iron supplementation (Table 4). This is astonishing since data in adult anemic IBD patients show that treatment is safe and well tolerated in most IBD patients either orally or intravenously and is associated with rapid improvement in clinical, hematological, and quality of life evaluations $[24,25]$. Such data does not exist for pediatric patients, but Goodhand et al. also reported that less children (13\%) and adolescents (30\%) with anemia and IBD received oral iron compared to adults (48\%) [9].

Pels et al. showed that expectant management of anemia in pediatric IBD patient leads to a slower hematologic recovery [15]. Persistent anemia in IBD patients is associated with fatigue and other impairments of health-related quality of life [26]. In this study, for the first time shown in a large cohort of pediatric IBD patients, general wellbeing, as measured by self-assessment, is significantly lower in anemic patients.

Treatment with iron supplementation seems mandatory for patients with proven iron deficiency anemia. For physicians from our registry considering all limitations, an undertreatment of highly prevalent microcytic anemia, potential iron deficient anemia has to be suspected. Possible causes are suspected side effects of iron supplementation orally or parenterally or a suspected cause for anemia in chronic inflammation. A possible explanation could be seen in the high number of patients with relatively mild anemia (less than $2 \mathrm{~g} / \mathrm{dl} \mathrm{Hb}$ below 3rd percentile), but even when looking at severe anemic patients alone, the proportion of patients that receive iron supplementation only increases slightly to $41 \%$ in UC, $22.2 \%$ in CD, and $20.4 \%$ in IBD-U.

The strength of this study is the large number of children and adolescents and the number of follow-up data from our pediatric IBD registry since well-powered studies and registry analysis are scarce in pediatric patients. Limitations are the collection method and study design, which is not tailored to reflect individual courses of disease but rather group characteristics with a cross-sectional instead of longitudinal approach, corresponding to the initial question. To answer questions about treatment and individual patients' benefit, a longitudinal approach has to be taken and is on the way in a future analysis.

\section{Conclusion}

There is a high prevalence of anemia in pediatric patients with IBD. Even more patients with $\mathrm{CD}$ are anemic than patients with UC. The rate is highest in adolescents at the onset of disease, and in young children, the rate of patients with severe anemia is higher. Anemia is associated with impairment in patient self-assessment of general well-being, physician assessment of disease activity, and higher disease activity as determined by activity indices. During the disease course, the prevalence of anemia remains high. In contrast, only one-third of patients are treated by iron supplementation. This undertreatment should be addressed by pediatric gastroenterologists.

\section{Additional Points}

Core Tip. About 2/3 of pediatric IBD patients are anemic at the time of diagnosis and during follow-up. Anemia correlates to disease severity and patients' self-assessment. In contrast, only about half of the patients receive iron therapy.

\section{Ethical Approval}

The database and reporting procedure were examined and approved by the relevant ethics committees and data protection employees of the three institutions that maintained the registry during the time period covered by the research (Institute for Medical Informatics and Biometry (IMB) of Technische Universität Dresden; Institute of Epidemiology at the Helmholtz Zentrum, LMU Munich; Department of General Pediatrics and Neonatology, JLU Giessen). The Austrian hospitals that participated also obtained local ethics approval.

\section{Consent}

Inclusion in the registry CEDATA-GPGE was only done after detailed information of the parents and depending on the age of the children and adolescents, too. All study participants, or their legal guardian, provided informed written consent prior to study enrollment. 


\section{Conflicts of Interest}

Martin W. Laass received speakers' fees from Vifor Pharma and Dr. Falk Pharma GmbH. Martin W. Laass took part in retrospective study on use of intravenous iron financed by Vifor Pharma. Stephan Buderus received speakers' fees from Dr. Falk Pharma GmbH, Abbvie, and Nestlé Nutrition Institute (NNI). The other authors have no conflicts of interest relevant to this article to disclose.

\section{Authors' Contributions}

Jan de Laffolie, Martin W. Laass, Dietmar Scholz, Klaus-Peter Zimmer, and Stephan Buderus were involved in the design of the registry and the current study. All authors including in the members of the study group participated in the data collection and their critical review and analysis. Jan de Laffolie and Dietmar Scholz performed the data extraction and statistical analysis; Jan de Laffolie, Martin W. Laass, Dietmar Scholz, Klaus-Peter Zimmer ${ }^{1}$, and Stephan Buderus $^{4}$ analyzed the data and results with respect to the current study objective; Jan de Laffolie wrote the draft of the paper, and Martin W. Laass and Stephan Buderus critically revised the manuscript for important intellectual content. Stephan Buderus and Jan de Laffolie finalized the manuscript.

\section{Acknowledgments}

The authors thank all members of the CEDATA-GPGE study group and the Saxonian Pediatric IBD registry for participating in the recruitment and our patients and their families. The authors greatly acknowledge the ongoing dedication and contribution for the technical assistance of the registry by $\mathrm{C}$. Wendt and T. Weidenhausen. The current member list of the core CEDATA-GPGE study group are as follows: Antje Ballauff (Krefeld, Germany), antje.ballauff@helioskliniken.de; Thomas Berger (Datteln, Germany), t.berger@kinderklinik-datteln.de; Stephan Buderus (Bonn, Germany), stephan.buderus@marien-hospital-bonn.de; Martin Claßen (Bremen, Germany), martin.classen@klinikum-bremen-ldw.de; Söhnke Dammann (Stuttgart, Germany), s.dammann@klinikum-stuttgart.de; Jan de Laffolie (Gießen, Germany), Jan.DeLaffolie@paediat.med.uni-giessen.de; Almuthe Hauer (Graz, Austria), almuthe.hauer@medunigraz.at; Klaus-Michael Keller (Wiesbaden, Germany), klaus-michael.keller@helioskliniken.de; Sibylle Koletzko (München, Germany), sibylle. koletzko@med.uni-muenchen.de; Andreas Krahl (Dortmund, Germany), andreas.krahl@kinderkliniken.de; Martin Laaß (Dresden, Germany), martin.laass@uniklinikum-dresden.de; Thomas Lang (Regensburg, Germany), thomas.lang@barmherzige-regensburg.de; Carsten Posovszky (Ulm, Germany), Carsten.Posovszky@uniklinik-ulm.de; Burkhard Rodeck (Osnabrück, Germany), b.rodeck@ckos.de; Stefan Trenkel (Potsdam, Germany), strenkel@klinikumevb.de; and KlausPeter Zimmer (Gießen, Germany), Klaus-Peter.Zimmer@paediat.med.uni-giessen.de. Vifor Pharma partially sponsored the study without influencing the design, data collection, and analysis or any part of the manuscript. The registry
CEDATA-GPGE has received financial support from the Falk-Foundation and the foundation "Ein Herz für Kinder" and the German Crohn's and Colitis Association "DCCV."

\section{References}

[1] N. A. Molodecky, I. S. Soon, D. M. Rabi et al., "Increasing incidence and prevalence of the inflammatory bowel diseases with time, based on systematic review," Gastroenterology, vol. 142, no. 1, pp. 46-54.e42, 2012.

[2] E. I. Benchimol, A. Guttmann, A. M. Griffiths et al., "Increasing incidence of paediatric inflammatory bowel disease in Ontario, Canada: evidence from health administrative data," Gut, vol. 58, pp. 1490-1497, 2009.

[3] E. I. Benchimol, K. J. Fortinsky, P. Gozdyra, M. Van den Heuvel, J. Van Limbergen, and A. M. Griffiths, "Epidemiology of pediatric inflammatory bowel disease: a systematic review of international trends," Inflammatory Bowel Diseases, vol. 17, no. 1, pp. 423-439, 2011.

[4] T. Longobardi, P. Jacobs, and C. N. Bernstein, "Work losses related to inflammatory bowel disease in the United States: results from the National Health Interview Survey," The American Journal of Gastroenterology, vol. 98, no. 5, pp. 1064-1072, 2003.

[5] A. Ferguson and D. M. Sedgwick, "Juvenile-onset inflammatory bowel disease: predictors of morbidity and health status in early adult life," Journal of the Royal College of Physicians of London, vol. 28, no. 3, pp. 220-227, 1994.

[6] M. D. Kappelman, K. R. Moore, J. K. Allen, and S. F. Cook, "Recent trends in the prevalence of Crohn's disease and ulcerative colitis in a commercially insured US population," Digestive Diseases and Sciences, vol. 58, no. 2, pp. 519-525, 2013.

[7] S. Buderus, D. Scholz, R. Behrens et al., "Inflammatory bowel disease in pediatric patients: characteristics of newly diagnosed patients from the CEDATA-GPGE registry," Deutsches Ärzteblatt International, vol. 112, no. 8, pp. 121-127, 2015.

[8] A. E. Wiskin, B. J. Fleming, S. A. Wootton, and R. M. Beattie, "Anaemia and iron deficiency in children with inflammatory bowel disease," Journal of Crohn's \& Colitis, vol. 6, no. 6, pp. 687-691, 2012.

[9] J. R. Goodhand, N. Kamperidis, A. Rao et al., "Prevalence and management of anemia in children, adolescents, and adults with inflammatory bowel disease," Inflammatory Bowel Diseases, vol. 18, no. 3, pp. 513-519, 2012.

[10] J. Van Limbergen, R. K. Russell, H. E. Drummond et al., "Definition of phenotypic characteristics of childhood-onset inflammatory bowel disease," Gastroenterology, vol. 135, no. 4, pp. 1114-1122, 2008.

[11] F. Gomollon and J. P. Gisbert, "Anemia and inflammatory bowel diseases," World Journal of Gastroenterology, vol. 15, no. 37, pp. 4659-4665, 2009.

[12] J. Ezri, P. Marques-Vidal, and A. Nydegger, "Impact of disease and treatments on growth and puberty of pediatric patients with inflammatory bowel disease," Digestion, vol. 85 , no. 4 , pp. 308-319, 2012.

[13] C. E. Brain and M. O. Savage, "Growth and puberty in chronic inflammatory bowel disease," Bailliere's Clinical Gastroenterology, vol. 8, no. 1, pp. 83-100, 1994.

[14] F. M. Ruemmele, G. Veres, K. L. Kolho et al., "Consensus guidelines of ECCO/ESPGHAN on the medical management 
of pediatric Crohn's disease," Journal of Crohn's \& Colitis, vol. 8, no. 10, pp. 1179-1207, 2014.

[15] L. P. Pels, E. Van de Vijver, H. J. Waalkens et al., "Slow hematological recovery in children with IBD-associated anemia in cases of "expectant management"," Journal of Pediatric Gastroenterology and Nutrition, vol. 51, no. 6, pp. 708-713, 2010.

[16] K. Gerasimidis, A. Barclay, A. Papangelou et al., "The epidemiology of anemia in pediatric inflammatory bowel disease: prevalence and associated factors at diagnosis and follow-up and the impact of exclusive enteral nutrition," Inflammatory Bowel Diseases, vol. 19, no. 11, pp. 24112422, 2013.

[17] W. Thierfelder, R. Dortschy, B. Hintzpeter, H. Kahl, and C. Scheidt-Nave, "Biochemical measures in the German Health Interview and Examination Survey for Children and Adolescents (KiGGS)," Bundesgesundheitsblatt, Gesundheitsforschung, Gesundheitsschutz, vol. 50, no. 5-6, pp. 757-770, 2007.

[18] J. S. Hyams, G. D. Ferry, F. S. Mandel et al., "Development and validation of a pediatric Crohn's disease activity index," Journal of Pediatric Gastroenterology and Nutrition, vol. 12, no. 4, pp. 439-447, 1991.

[19] D. Turner, A. R. Otley, D. Mack et al., "Development, validation, and evaluation of a pediatric ulcerative colitis activity index: a prospective multicenter study," Gastroenterology, vol. 133, no. 2, pp. 423-432, 2007.

[20] A. Timmer, R. Behrens, S. Buderus et al., "Childhood onset inflammatory bowel disease: predictors of delayed diagnosis from the CEDATA German-language pediatric inflammatory bowel disease registry," The Journal of Pediatrics, vol. 158, no. 3, pp. 467-473.e2, 2011.

[21] M. Thayu and P. Mamula, "Treatment of iron deficiency anemia in pediatric inflammatory bowel disease," Current Treatment Options in Gastroenterology, vol. 8, no. 5, pp. 411417, 2005.

[22] J. Belaiche, J. P. Desager, Y. Horsmans, and E. Louis, "Therapeutic drug monitoring of azathioprine and 6mercaptopurine metabolites in Crohn disease," Scandinavian Journal of Gastroenterology, vol. 36, pp. 71-76, 2001.

[23] C. Cuffari, Y. Theoret, S. Latour, and G. Seidman, "6-Mercaptopurine metabolism in Crohn's disease: correlation with efficacy and toxicity," Gut, vol. 39, pp. 401-406, 1996.

[24] J. P. Gisbert, F. Bermejo, R. Pajares et al., "Oral and intravenous iron treatment in inflammatory bowel disease: hematological response and quality of life improvement," Inflammatory Bowel Diseases, vol. 15, no. 10, pp. 1485-1491, 2009.

[25] M. W. Laass, D. Roggenbuck, and K. Conrad, "Diagnosis and classification of Crohn's disease," Autoimmunity Reviews, vol. 13, pp. 467-471, 2014.

[26] M. J. Romberg-Camps, Y. Bol, P. C. Dagnelie et al., "Fatigue and health-related quality of life in inflammatory bowel disease: results from a population-based study in the Netherlands: the IBD-South Limburg cohort," Inflammatory Bowel Diseases, vol. 16, no. 12, pp. 2137-2147, 2010. 




The Scientific World Journal
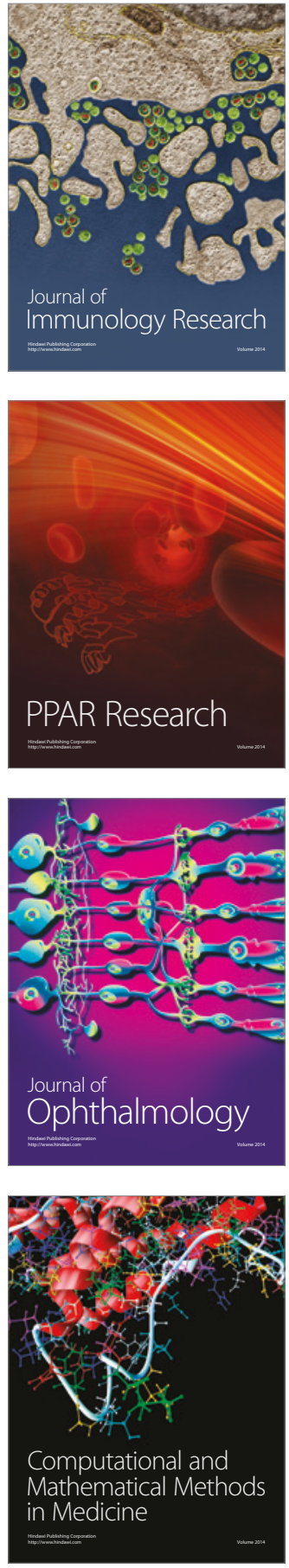

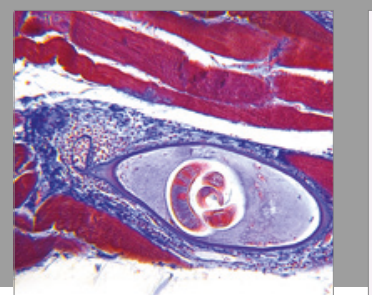

Gastroenterology Research and Practice


\section{Hindawi}

Submit your manuscripts at

https://www.hindawi.com
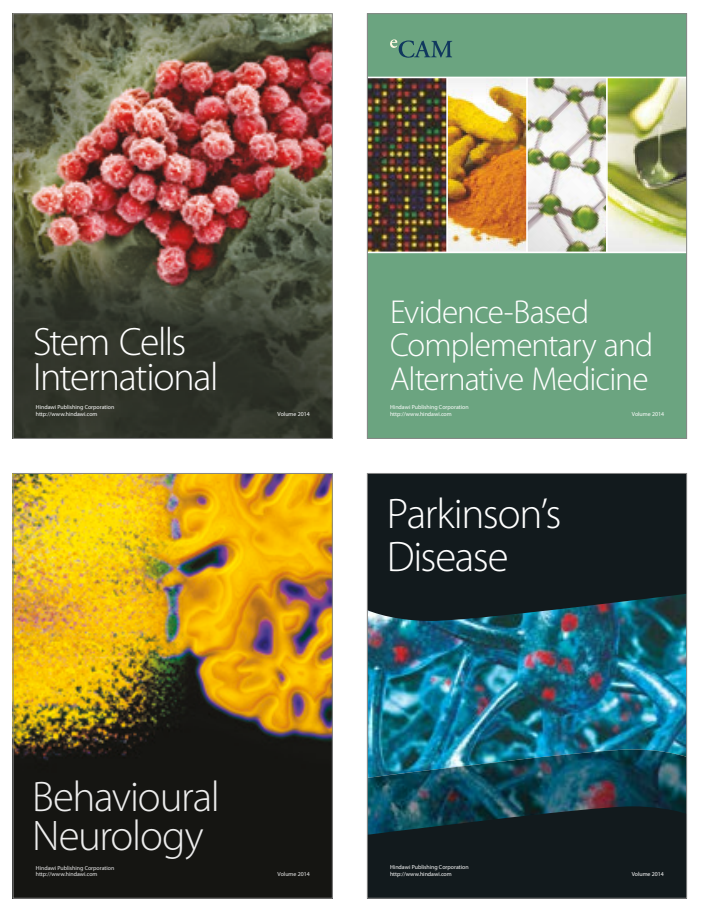
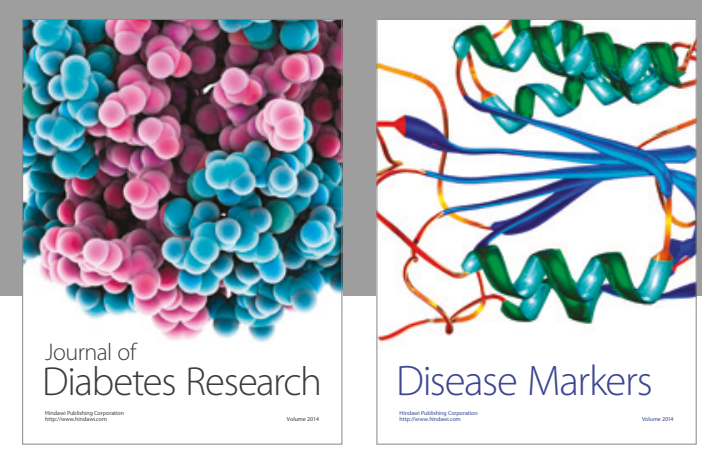

Disease Markers
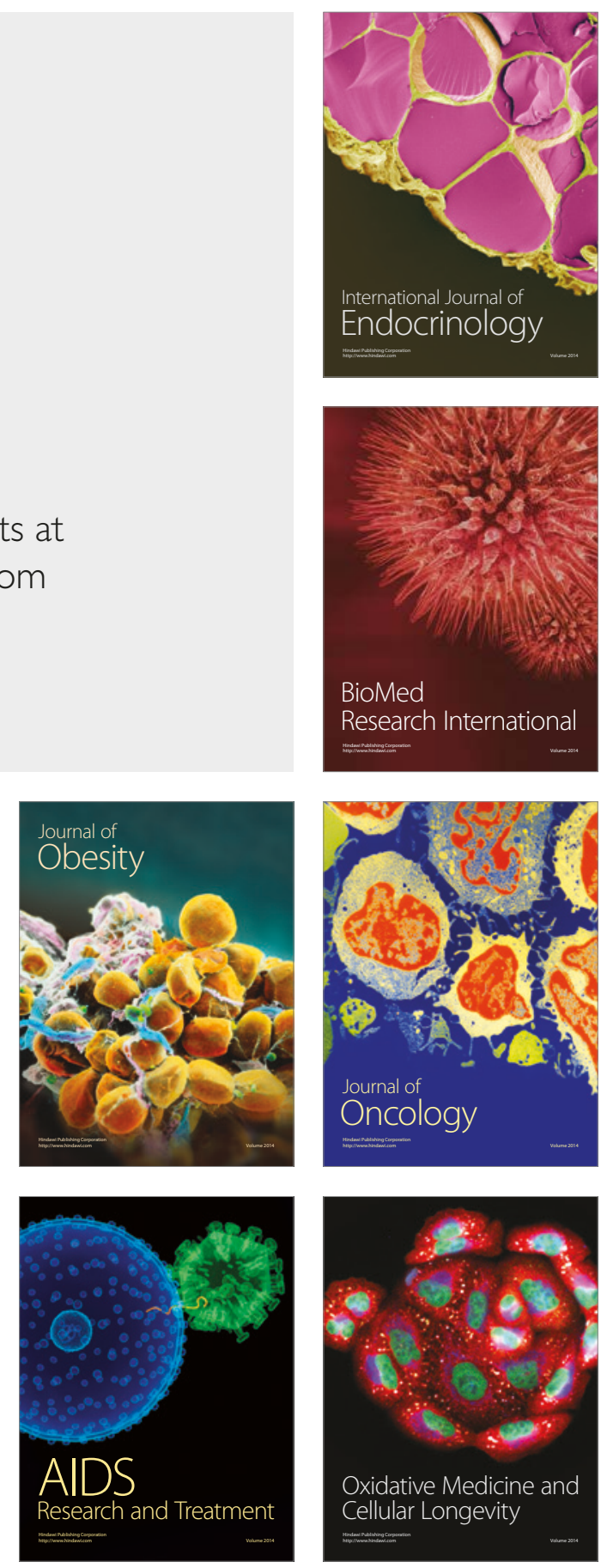